\title{
Hydrodynamic Coupling of Two Brownian Spheres to a Planar Surface
}

\author{
Eric R. Dufresne, ${ }^{1}$ Todd M. Squires, ${ }^{2}$ Michael P. Brenner, ${ }^{3}$ and David G. Grier ${ }^{1}$ \\ ${ }^{1}$ Department of Physics, James Franck Institute, and Institute for Biophysical Dynamics, The University of Chicago, \\ Chicago, Illinois 60637 \\ ${ }^{2}$ Department of Physics, Harvard University, Cambridge, Massachusetts 02138 \\ ${ }^{3}$ Department of Mathematics, Massachusetts Institute of Technology, Cambridge, Massachusetts 02139
}

(Received 22 March 2000)

\begin{abstract}
We describe direct imaging measurements of the collective and relative diffusion of two colloidal spheres near a flat plate. The bounding surface modifies the spheres' dynamics, even at separations of tens of radii. This behavior is captured by a stokeslet analysis of fluid flow driven by the spheres' and wall's no-slip boundary conditions. In particular, this analysis reveals surprising asymmetry in the normal modes for pair diffusion near a flat surface.
\end{abstract}

PACS numbers: 82.70.Dd, 66.10.Cb, 67.40.Hf

Despite considerable progress over the past two centuries [1], hydrodynamic properties of all but the simplest colloidal systems remain controversial or unexplained. For example, velocity fluctuations in sedimenting colloidal suspensions are predicted to diverge with system size [2]. Experimental observations indicate, on the other hand, that long-wavelength fluctuations are suppressed by an as-yet undiscovered mechanism [3]. One possible explanation is that hydrodynamic coupling to bounding surfaces may influence particles' motions to a greater extent and over a longer range than previously suspected [4]. Such considerations invite a renewed examination of how hydrodynamic coupling to bounding surfaces influences colloidal particles' dynamics.

This Letter describes an experimental and theoretical investigation of two colloidal spheres' diffusion near a flat plate. Related studies have addressed the dynamics of two spheres far from bounding walls [5], and of a single sphere in the presence of one [6] or two walls $[7,8]$. Confinement by two walls poses particular difficulties since available theoretical predictions apply only for highly symmetric arrangements [9], or else contradict each other $[8,10]$. The geometry we have chosen avoids some of this complexity while highlighting the range of nonadditive hydrodynamic coupling in confined colloidal suspensions.

We combined optical tweezer manipulation [11] and digital video microscopy [12] to measure four components of the pair diffusion tensor for two colloidal spheres as a function of their center-to-center separation $r$ and of their height $h$ above a flat glass surface. Measurements were performed on silica spheres of radius $0.495 \pm 0.025 \mu \mathrm{m}$ (Duke Scientific lot 21024) dispersed in a layer of water $140 \pm 2 \mu \mathrm{m}$. The suspension was sandwiched between a microscope slide and a No. 1 coverslip whose surfaces were stringently cleaned before assembly [13] and whose edges were hermetically sealed with a uv cured epoxy (Norland type 88) to prevent evaporation and suppress bulk fluid flow. A transparent thin film heater bonded to the microscope slide and driven by a Lakeshore LM-330 temperature controller maintained the sample volume's temperature at $T=29.00 \pm 0.01{ }^{\circ} \mathrm{C}, \quad$ as measured by a platinum resistance thermometer. The addition of $2 \mathrm{mM}$ of $\mathrm{NaCl}$ to the solution minimized electrostatic interactions among the weakly charged spheres and glass surfaces by reducing the Debye screening length to $7 \mathrm{~nm}$. Under these conditions, the individual spheres' free self-diffusion coefficients are expected to be $D_{0}=k_{B} T /(6 \pi \eta a)=0.550 \pm 0.028 \mu \mathrm{m}^{2} / \mathrm{s}$, where $\eta=0.817 \mathrm{cP}$ is the electrolyte's viscosity [14].

The spheres' motions were tracked with an Olympus IMT-2 optical microscope using a $100 \times$ NA 1.4 oil immersion objective. Images acquired with an NEC TI-324A CCD camera were recorded on a JVC-S822DXU SVHS video deck before being digitized with a Mutech MV-1350 frame grabber at $1 / 60 \mathrm{~s}$ intervals. Field-accurate digitization was assured by interpreting the vertical interlace time code recorded onto each video field. The spheres' locations $\vec{r}_{1}(t)$ and $\vec{r}_{2}(t)$ in the image acquired at time $t$ then were measured to within $20 \mathrm{~nm}$ using a computerized centroid tracking algorithm [12].

A pair of spheres was placed reproducibly in a plane parallel to the glass surfaces using optical tweezers [11]. These optical traps were created with a solid state laser (Coherent Verdi) whose beam was brought to focus within the sample volume by the microscope's objective. Resulting optical gradient forces sufficed to localize a silica sphere at the focal point despite random thermal forces [11]. Two optical traps were created by alternating the focused laser spot between two positions in the focal plane at $200 \mathrm{~Hz}$ using a galvanometer-driven mirror [15]. Diverting the trapping laser onto a beam block every few cycles freed the spheres to diffuse away from this well-defined initial condition. Resuming the trap's oscillation between the two trapping points reset the spheres' positions. Alternately trapping and releasing the spheres allowed us to sample their dynamics efficiently in a range of configurations. Allowing the spheres only $t=83 \mathrm{~ms}$ (five video fields) of freedom before retrapping them for $16 \mathrm{~ms}$ (less than one video field) ensured that their out-of-plane 
diffusion, $\Delta z<\sqrt{2 D_{0} t}=0.3 \mu \mathrm{m}$, caused negligible tracking errors.

Because optical tweezers form in the microscope's focal plane, their height $h$ relative to the coverslip's surface can be adjusted from 1 to $30 \mu \mathrm{m}$ with $0.5 \mu \mathrm{m}$ accuracy by adjusting the microscope's focus. For a given height, we continuously varied the spheres' initial separation between 2 and $10 \mu \mathrm{m}$ at $0.025 \mathrm{~Hz}$ for a total of $20 \mathrm{~min}$. This procedure yielded 60000 samples of the spheres' dynamics in $1 / 60 \mathrm{~s}$ intervals divided into sequences $5 / 60 \mathrm{~s}$ long for each value of $h$. These trajectories were decomposed into cooperative motions $\vec{\rho}=\vec{r}_{1}+\vec{r}_{2}$ and relative motions $\vec{r}=\vec{r}_{1}-\vec{r}_{2}$ either perpendicular or parallel to the initial separation vector, and binned according to the initial separation, $r$. The diffusion coefficients $D_{\psi}(r, h)$ associated with each mode of motion $\psi(r, h, \tau)$ were then obtained from

$$
\left\langle\Delta \psi^{2}(\tau)\right\rangle=2 D_{\psi}(r, h) \tau,
$$

where the angle brackets indicate an ensemble average.

Figure 1 shows typical data for one mode of motion at one height and starting separation. Diffusion coefficients extracted from least squares fits to Eq. (1) appear in Fig. 2 as functions of $r$ for the smallest and largest accessible values of $h$. In the absence of other interactions, the observed trends reflect hydrodynamic coupling between the spheres and the bounding surface.

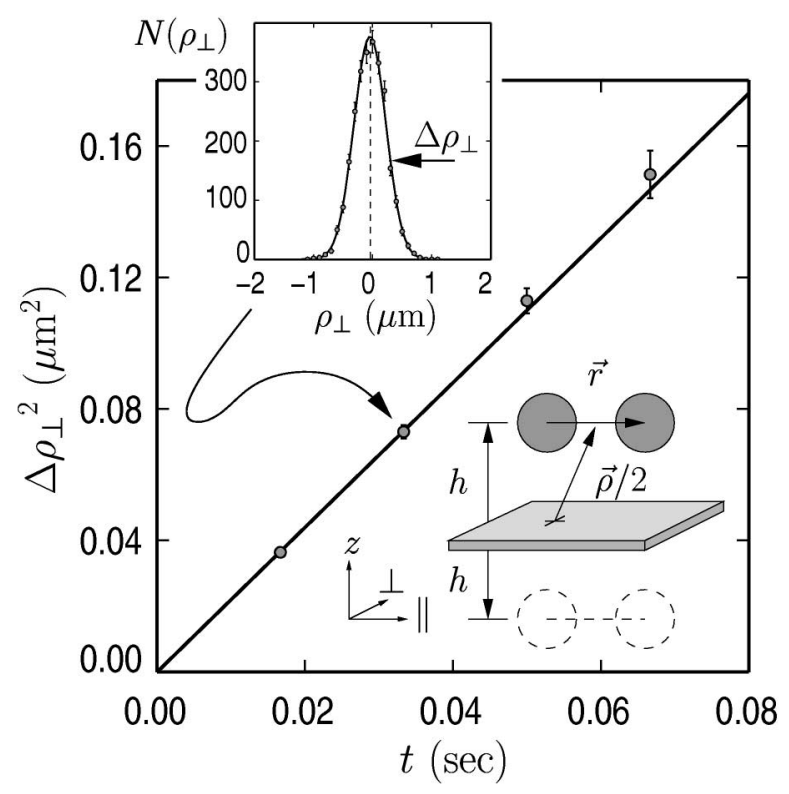

FIG. 1. Measuring pair diffusion for the geometry depicted in the lower inset. Dashed spheres represent hydrodynamic images. The upper inset shows the histogram of two spheres' collective displacements in the $\perp$ direction starting from $h=$ $25.5 \pm 0.7 \mu \mathrm{m}, r=7.00 \pm 0.25 \mu \mathrm{m}$, and $\rho=0$, after free diffusion for $\tau=1 / 30 \mathrm{~s}$. Fitting to a Gaussian yields the rms displacement $\Delta \rho_{\perp}(r, h, \tau)$. The main plot tracks the evolution of $\Delta \rho_{\perp}^{2}(r, h, \tau)$ together with a least squares fit to Eq. (1) for the diffusion coefficient $D_{\perp}^{C}(r, h)$. The result is indicated by an arrow in Fig. 2(d).
Particles moving through a fluid excite large-scale flows through the no-slip boundary condition at their surfaces. These flows couple distant particles' motions, so that each particle's dynamics depends on the particular configuration of the entire collection. This dependence is readily calculated using Batchelor's generalization of Einstein's classic argument [16]: The probability to find $N$ particles at equilibrium in a particular configuration $\left\{\vec{r}_{1}, \ldots, \vec{r}_{N}\right\}$ depends on their interaction $\Phi\left(\vec{r}_{1}, \ldots, \vec{r}_{N}\right)$ through Boltzmann's distribution, $P\left(\vec{r}_{1}, \ldots, \vec{r}_{N}\right)=\exp \left[-\Phi /\left(k_{B} T\right)\right]$. The corresponding force $-\nabla \Phi=k_{B} T \nabla P / P$ drives a probability flux $k_{B} T \mathbf{b} \nabla P$, where $\mathbf{b}\left(\vec{r}_{1}, \ldots, \vec{r}_{N}\right)$ is the particles' mobility tensor. The system reaches equilibrium when this interaction-driven flux is balanced by a diffusive flux $-\mathbf{D} \nabla P$. It follows that the $N$-particle diffusivity is $\mathbf{D}=k_{B} T \mathbf{b}$. Elements of $\mathbf{D}$ parametrize generalized diffusion relations [17]

$$
\left\langle\Delta r_{i \alpha}(\tau) \Delta r_{j \beta}(\tau)\right\rangle=2 D_{i \alpha, j \beta} \tau,
$$

describing how particle $i$ 's motion in the $\alpha$ direction couples to particle $j$ 's in the $\beta$ direction.

To first order in the particle radius, the mobility tensor for spheres of radius $a$ has the form

$$
b_{i \alpha, j \beta}=\frac{\delta_{i \alpha, j \beta}}{6 \pi \eta a}+b_{i \alpha, j \beta}^{e},
$$
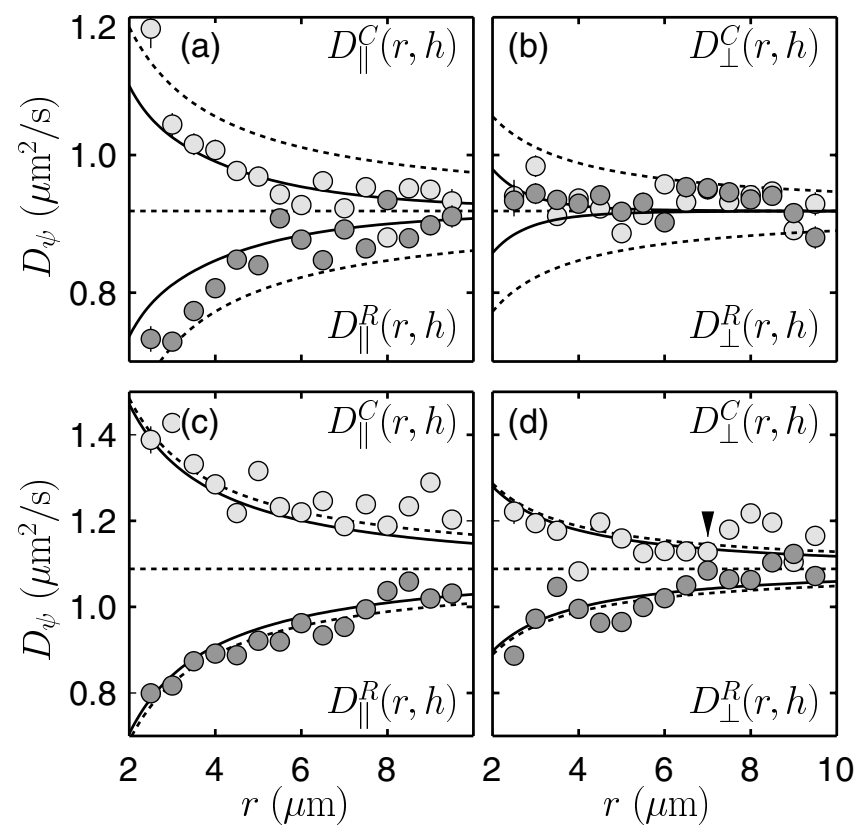

FIG. 2. Pair diffusion coefficients for $1 \mu \mathrm{m}$ diameter silica spheres as a function of center-to-center separation $r$ and at two different center-to-surface heights $h$. (a),(b) $h=$ $1.55 \pm 0.66 \mu \mathrm{m}$. (c),(d) $h=25.5 \pm 0.7 \mu \mathrm{m}$. (a),(c) Collective and relative motions parallel to the initial separation vector. (b),(d) Perpendicular. Dashed curves result from linear superposition of drag coefficients. Solid curves result from the theory described here, with no adjustable parameters. Horizontal dashed lines indicate the asymptotic self-diffusion coefficient $D_{x y}(h)$ from Eq. (11). 
where $b_{i \alpha, j \beta}^{e}$ describes the flow at $\vec{r}_{i}$ in the $\alpha$ direction due to an external force acting on a sphere at $\vec{r}_{j}$ in the $\beta$ direction. In the present discussion, it accounts for no-slip boundary conditions at all other surfaces in the system.

If the spheres are well separated, we may approximate the flow field around a given sphere by a stokeslet, the flow due to a unit point force at the sphere's location. The Green's function for the flow at $\vec{x}$ in the $\alpha$ direction due to a unit force at $\vec{r}_{j}$ in the $\beta$ direction is [18]

$$
\begin{aligned}
G_{\alpha \beta}^{S}\left(\vec{x}-\vec{r}_{j}\right)=\frac{1}{8 \pi \eta} & {\left[\frac{\delta_{\alpha \beta}}{\left|\vec{x}-\vec{r}_{j}\right|}\right.} \\
& \left.+\frac{\left(\vec{x}-\vec{r}_{j}\right)_{\alpha}\left(\vec{x}-\vec{r}_{j}\right)_{\beta}}{\left|\vec{x}-\vec{r}_{j}\right|^{3}}\right],
\end{aligned}
$$

so that $b_{i \alpha, j \beta}^{e}=G_{\alpha \beta}^{S}\left(\vec{r}_{i}-\vec{r}_{j}\right)$. In the particular case of two identical spheres, diagonalizing the resulting diffusivity tensor $\mathbf{D}$ yields the diffusion coefficients for two collective $(C)$ modes and two relative $(R)$ modes along directions perpendicular $(\perp)$ and parallel $(\|)$ to the initial separation [16]:

$$
\begin{aligned}
& \frac{D_{\perp}^{C, R}(r)}{2 D_{0}}=1 \pm \frac{3}{4} \frac{a}{r}+\mathcal{O}\left(\frac{a^{3}}{r^{3}}\right), \\
& \frac{D_{\|}^{C, R}(r)}{2 D_{0}}=1 \pm \frac{3}{2} \frac{a}{r}+\mathcal{O}\left(\frac{a^{3}}{r^{3}}\right),
\end{aligned}
$$

where the positive corrections apply to collective modes and the negative to relative. The collective diffusion coefficients $D_{\perp}^{C}$ and $D_{\|}^{C}$ are enhanced by hydrodynamic coupling because fluid displaced by one sphere entrains the other. Relative diffusion coefficients $D_{\perp}^{R}$ and $D_{\|}^{R}$ are suppressed, on the other hand, by the need to transport fluid into and out of the space between the spheres.

Introducing a planar boundary adds considerable complexity. The flow field around a small sphere located a height $h$ above a horizontal wall is most easily calculated by the method of images [19], in which the wall's no-slip boundary condition is satisfied by placing a stokeslet (S), a source doublet (D), and a stokeslet doublet (SD) a distance $h$ below the plane of the wall $[18,19]$. The flow due to this image system is described by the Green's function

$$
\begin{aligned}
G_{\alpha \beta}^{W}\left(\vec{x}-\vec{R}_{j}\right)= & -G_{\alpha \beta}^{S}\left(\vec{x}-\vec{R}_{j}\right)+2 h^{2} G_{\alpha \beta}^{D}\left(\vec{x}-\vec{R}_{j}\right) \\
& -2 h G_{\alpha \beta}^{\mathrm{SD}}\left(\vec{x}-\vec{R}_{j}\right),
\end{aligned}
$$

where $\vec{R}_{j}=\vec{r}_{j}-2 h \hat{z}$ is the position of sphere $j$ 's image, and

$$
\begin{gathered}
G_{\alpha \beta}^{D}(\vec{y})=\frac{1}{8 \pi \eta}\left(1-2 \delta_{\beta z}\right) \frac{\partial}{\partial y_{\beta}}\left(\frac{y_{\alpha}}{y^{3}}\right), \\
G_{\alpha \beta}^{\mathrm{SD}}(\vec{y})=\left(1-2 \delta_{\beta z}\right) \frac{\partial}{\partial y_{\beta}} G_{\alpha z}^{S}(\vec{y})
\end{gathered}
$$

are Green's functions for a source dipole and a stokeslet doublet, respectively. The flow field set up by the image system (and thus by the wall's no-slip boundary condition) entrains the sphere through $b_{i \alpha, i \beta}^{e}=G_{\alpha \beta}^{W}\left(\vec{r}_{i}-\vec{R}_{i}\right)$ and decreases its mobility. Two independent modes emerge from this analysis, one $(z)$ normal to the wall and the other (xy) parallel, with diffusivities [9]

$$
\begin{gathered}
\frac{D_{z}(h)}{D_{0}}=1-\frac{9}{8} \frac{a}{h}+\mathcal{O}\left(\frac{a^{3}}{h^{3}}\right), \\
\frac{D_{x y}(h)}{D_{0}}=1-\frac{9}{16} \frac{a}{h}+\mathcal{O}\left(\frac{a^{3}}{h^{3}}\right) .
\end{gathered}
$$

Equations (5) and (6) should suffice for two spheres far from bounding surfaces. Similarly, the spheres' motions should decouple when the influence of a nearby wall dominates; Eqs. (10) and (11) then should apply. At intermediate separations, however, neither set is accurate. Naively adding the drag coefficients [9] due to sphere-sphere and sphere-wall interactions yields $D_{\psi}^{-1}(r, h)=D_{\psi}^{-1}(r)+$ $\left[D_{x y}^{-1}(h)-D_{0}^{-1}\right] / 2$. Results of this linear superposition approximation appear as dashed curves in Fig. 2. While adequate for spheres more than 50 radii from the wall, linear superposition underestimates the wall's influence for smaller separations.

A more complete treatment not only resolves these quantitative discrepancies but also reveals an additional influence of the bounding surface on the spheres' dynamics: the highly symmetric and experimentally accessible modes parallel to the wall are no longer independent.

Each sphere interacts with its own image, its neighbor, and its neighbor's image. These influences contribute $b_{i \alpha, j \beta}^{e}=\left(1-\delta_{i j}\right) G_{\alpha \beta}^{S}\left(\vec{r}_{i}-\vec{r}_{j}\right)+G_{\alpha \beta}^{W}\left(\vec{r}_{i}-\vec{R}_{j}\right)$ to the mobility of sphere $i$ in the $\alpha$ direction. Eigenvectors of the corresponding diffusivity tensor appear in Fig. 3. The independent modes of motion are rotated with respect to the bounding wall by an amount which depends strongly on both $r$ and $h$. Even though the experimentally measured in-plane motions are not independent, they still satisfy

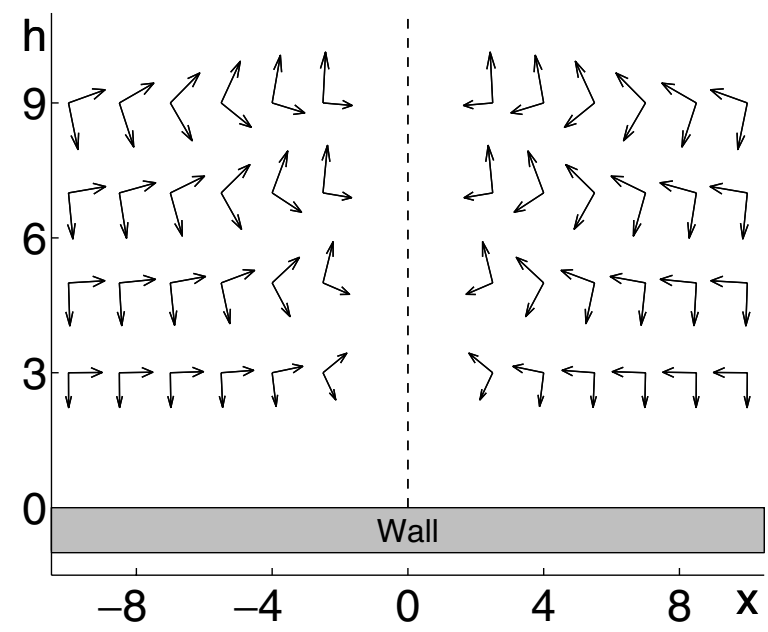

FIG. 3. Cross-sectional view of the diffusive modes for two spheres near a wall. Collective motion normal to the wall becomes increasingly coupled with relative motion parallel to the wall as $h$ approaches $r$. Collective normal modes at large $r$ cross over continuously to relative parallel modes as $r$ decreases. The dashed line at $x=0$ indicates the symmetry plane. 


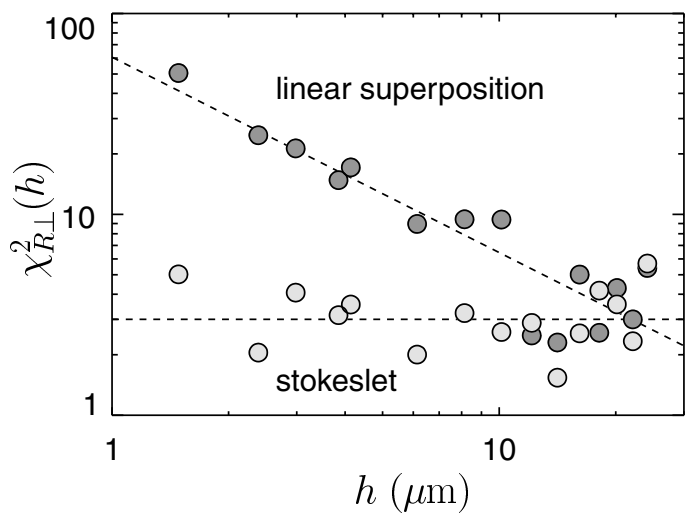

FIG. 4. Mean-squared deviations between measured and predicted diffusion coefficients for relative perpendicular motion, averaged over initial separations $r$. Dashed lines are guides to the eye and emphasize the $a / h$ leading-order error in the linear superposition model's predictions.

Eq. (2) with pair-diffusion coefficients $D_{\alpha}^{C, R}(r, h)=$ $D_{1 \alpha, 1 \alpha}(r, h) \pm D_{1 \alpha, 2 \alpha}(r, h)$, where the positive sign corresponds to collective motion, the negative to relative motion, and $\alpha$ indicates directions either perpendicular or parallel to the line connecting the spheres' centers. Explicitly, we obtain

$$
\frac{D_{\perp}^{C, R}(r, h)}{2 D_{0}}=1-\frac{9}{16} \frac{a}{h} \pm \frac{3}{4} \frac{a}{r}\left[1-\frac{1+\frac{3}{2} \xi}{(1+\xi)^{3 / 2}}\right],
$$

$$
\begin{aligned}
\frac{D_{\|}^{C, R}(r, h)}{2 D_{0}} & =1-\frac{9}{16} \frac{a}{h} \\
& \pm \frac{3}{2} \frac{a}{r}\left[1-\frac{1+\xi+\frac{3}{2} \xi^{2}}{(1+\xi)^{5 / 2}}\right]
\end{aligned}
$$

up to $\mathcal{O}\left(a^{3} / r^{3}\right)$ and $\mathcal{O}\left(a^{3} / h^{3}\right)$, where $\xi=4 h^{2} / r^{2}$. These results appear as solid curves in Fig. 2.

To gauge the success of this procedure and to quantify the range over which the presence of a wall measurably influences colloidal dynamics, we computed the error-weighted mean-squared deviation of the predicted diffusivities from the measured values, $\chi_{\psi}^{2}(h)=\frac{1}{N} \sum_{i=1}^{N} \sigma_{i}^{-2}\left[D_{\psi}^{m}\left(r_{i}, h\right)-D_{\psi}\left(r_{i}, h\right)\right]^{2}$, where $D_{\psi}^{m}\left(r_{i}, h\right)$ is the measured diffusivity of mode $\psi$ at $r_{i}$ and $h$, and $\sigma_{i}$ is the statistical uncertainty in $D_{\psi}^{m}\left(r_{i}, h\right)$. Typical results appear in Fig. 4. Predictions based on the stokeslet approximation agree well with measurement over the entire experimentally accessible range. Deviations from the linear superposition approximation's predictions, on the other hand, are evident to $h=15 \mu \mathrm{m}$ or 30 radii.

The present study demonstrates that a confining surface can influence colloidal dynamics even at large separations, and that this three-surface coupling is accurately described by a leading-order stokeslet approximation. This success suggests that the same formalism can be applied to more general configurations of spheres and bounding surfaces. Our results also reveal that wall-induced hydrodynamic interactions may have influenced nonequilibrium optical tweezer measurements of confined colloidal interactions [20], and could have contributed to the observed attractions between like-charged spheres [21]. This possibility is explored elsewhere [22].

Work at the University of Chicago was supported by the National Science Foundation through Grant No. DMR-9730189, through the MRSEC program of the NSF through Grant No. DMR-9880595, and by the David and Lucile Packard Foundation. Theoretical work was supported by the A. P. Sloan Foundation, the Mathematical Science Division of the National Science Foundation, and a NDSEG grant to T. S.

[1] T. G. M. van de Ven, Colloidal Hydrodynamics (Academic Press, San Diego, 1989).

[2] R.E. Caflisch and J.H.C. Luke, Phys. Fluids 28, 759 (1985).

[3] J.-Z. Xue et al., Phys. Rev. Lett. 69, 1715 (1992); H. Nicolai and E. Guazzelli, Phys. Fluids 7, 3 (1995); P. N. Segrè, E. Herbolzheimer, and P. M. Chaikin, Phys. Rev. Lett. 79, 2574 (1997).

[4] M. P. Brenner, Phys. Fluids 11, 754 (1999).

[5] J. Crocker, J. Chem. Phys. 106, 2837 (1997); J.-C. Meiners and S. R. Quake, Phys. Rev. Lett. 82, 2211 (1999).

[6] A. Pralle, E.-L. Florin, E. H. K. Stelzer, and J. K. H. Hörber, Appl. Phys. A 66, S71 (1998).

[7] L. P. Faucheux and A. J. Libchaber, Phys. Rev. E 49, 5158 (1994); B. Lin, J. Yu, and S. A. Rice, Phys. Rev. E (to be published).

[8] L. Lobry and N. Ostrowsky, Phys. Rev. B 53, 12050 (1996).

[9] J. Happel and H. Brenner, Low Reynolds Number Hydrodynamics (Kluwer, Dordrecht, 1991).

[10] N. Liron and S. Mochon, J. Eng. Math. 10, 287 (1976).

[11] D. G. Grier, Curr. Opin. Colloid Interface Sci. 2, 264 (1997).

[12] J. C. Crocker and D. G. Grier, J. Colloid Interface Sci. 179, 298 (1996).

[13] M.L. Hair, in Clean Surfaces, edited by G. Goldfinger (Dekker, New York, 1970), pp. 269-284.

[14] D. S. Viswanath and G. Natarajan, Data Book on the Viscosity of Liquids (Hemisphere, New York, 1989).

[15] K. Sasaki et al., Opt. Lett. 16, 1463 (1991).

[16] G. K. Batchelor, J. Fluid Mech. 74, 1 (1976).

[17] D. L. Ermak and J. A. McCammon, J. Chem. Phys. 69, 1352 (1978).

[18] C. Pozrikidis, Boundary Integral and Singularity Methods for Linearized Viscous Flow (Cambridge University Press, New York, 1992).

[19] J. R. Blake, Prog. Colloid Polym. Sci. 70, 303 (1971).

[20] J. C. Crocker and D. G. Grier, Phys. Rev. Lett. 77, 1897 (1996).

[21] A.E. Larsen and D. G. Grier, Nature (London) 385, 230 (1997).

[22] T. M. Squires and M. P. Brenner, cond-mat/0003195. 